\title{
Evaluación entre compañeros de la comunicación oral efectiva
}

\author{
José M. Meseguer-Dueñas ${ }^{a}$, Ana Vidaurreb ${ }^{b}$ José Molina-Mateo $^{c}$, Jaime Riera Guasp ${ }^{d}$,
} Rosa Martínez Sala ${ }^{\mathrm{e}}$.

Departamento de Física Aplicada. Escuela Técnica Superior de Ingeniería del Diseño. Universitat Politècnica de València

ajmmesegu@fis.upv.es, ${ }^{b}$ vidaurre@fis.upv.es, ${ }^{c}$ jmmateo@fis.upv.es, ${ }^{\text {j }}$ jriera@fis.upv.es, ermsala@fis.upv.es

\begin{abstract}
Peer assessment is a form of collaborative learning where students evaluate the product of learning of other students. In our case, students perform two types of evaluation related to effective communication: they evaluate quantitatively, in teamwork, problems solved by another team, and later they evaluate qualitatively oral presentations of problem solutions. They have been given guidelines on how to perform the evaluation. The result has been compared to the assessment made by teachers. In the qualitative assessment of the oral presentations, we have not found significant differences between students and professors. Furthermore, the arguments that support evaluations are rigorous and show their learning through work done by peers.
\end{abstract}

Keywords: collaborative learning, work team, effective communication, peer review.

\begin{abstract}
Resumen
La evaluación entre compañeros es una forma de aprendizaje colaborativo en el que los estudiantes valoran el producto de aprendizaje de otros estudiantes. En nuestro caso, efectúan dos tipos de evaluación relacionados con la comunicación efectiva: en equipo evalúan cuantitativamente documentos con la resolución de problemas de otros equipos, y, posteriormente, evalúan cualitativamente las presentaciones orales de los ejercicios. Se les han dado pautas sobre como llevar a cabo la evaluación. El resultado ha sido comparado con la evaluación hecha por los profesores. En la evaluación cualitativa de las presentacions no se ha encontrado diferencias significativas entre la realizada por los alumnos en equipo y la de los profesores. Además, la argumentación que hacen a las valoraciones son rigurosas y muestran su aprendizaje a través del trabajo hecho por los compañeros.
\end{abstract}

Palabras clave: aprendizaje colaborativo, trabajo en equipo, comunicación efectiva, evaluación entre compañeros. 


\section{Introducción}

Desarrollar capacidades en los estudiantes para cooperar, comunicarse y tomar decisiónes es una tarea crucial en el desarrollo de los actuales planes de estudio. La evaluación entre pares permite a los estudiantes observar el trabajo de otros, preguntarse cuestiones y dirigir la discusión, lo que conduce a mejorar el pensamiento crítico y a tener una mejor percepción de su propio trabajo (Black \& Wiliam, 1998), (Shepard, 2000), (Lin, Liu, \& Yuan, 2001). Cuando los trabajos son evaluados por los compañeros de forma crítica y constructiva se consigue una mejora de ambos actores: los que evalúan y los que son evaluados (C.-C. Tsai, Lin, \& Yuan, 2002). Varios autores han demostrado que la calidad de los trabajos mejora cuando los estudiantes reciben feedback de sus compañeros (Cheng, Hou, \& Wu, 2014) (Prins, Sluijsmans, Kirschner, \& Strijbos, 2005), (C. C. Tsai \& Liang, 2009), (Tseng \& Tsai, 2007).

La opinión de los estudiantes que son evaluados por sus pares no es unánime, encontrándose algunas actitudes negativas, lo que se expresa en términos de dudas respecto a las capacidades de "los pares", o sensación de injusticia (Levine, Kelly, Karakoc, \& Haidet, 2007), (Kaufman \& Schunn, 2011) (Wen \& Tsai, 2006). Sea positivo o negativo, el juicio de los compañeros siempre evoca una respuesta emocional (N Falchikov, 2005), (Sargeant, Mann, Sinclair, Van Der Vleuten, \& Metsemakers, 2008). En este sentido, el docente debe ser capaz de comprender y gestionar las posibles consecuencias. La evaluación entre pares debe ser expuesta y comprendida como una opción de mejora más que una crítica al trabajo o a la persona (Casamayor, Amandi, \& Campo, 2009). Weiss (Weiss, 2000) sugiere que las emociones pueden estimular la atención y ésta estimular el aprendizaje.

Algunos profesores son reticentes a utilizar la evaluación entre pares porque dudan de su validez. Es decir, no confían en que las puntuaciones de los estudiantes coincidan con sus puntuaciones (N. Falchikov \& Goldfinch, 2000), (Ibarra Sáiz, Gómez Rodríguez, \& Gómez Ruiz, 2012). Sin embargo, las puntuaciones asignadas a un producto (examen, informe de laboratorio, resolución de un problema,...) varían de un profesor a otro. Y, aun cuando coincidan las puntuaciones asignadas por varios profesores, hay muchos trabajos que plantean dudas sobre su validez; ya que éstas, "puntuaciones consistentes" no implican necesariamente "puntuaciones justas" (Nancy Falchikov \& Magin, 1997), (Marcoulides \& Simkin, 1991). Falchikov (N. Falchikov \& Goldfinch, 2000) concluye que cuando se utiliza la evaluación entre compañeros es preferible hacerlo en un contexto de evaluación de productos académicos, en pequeños grupos, y proporcionando una valoración global, con criterios bien entendidos y consensuados por todos. La evaluación entre compañeros, obtiene los máximos beneficios cuando es acompañada de comentarios razonados y de interacción entre los estudiantes (tanto entre los que juzgan, como entre los que juzgan y son juzgados) (Liu \& Carless, 2006).

Aunque el trabajo en equipo ha sido utilizado habitualmente como parte de las metodologías de aprendizaje, es recientemente cuando se ha prestado especial atención a la evaluación de la competencia en sí, además de los productos (memorias de laboratorio, por ejemplo). En este contexto, la evaluación orientada al aprendizaje, en general, y la

(cc) BY-NC-ND 2016, Universitat Politècnica de València 
autoevaluación y evaluación por pares, en particular, juegan un papel fundamental, de cara a desarrollar el pensamiento crítico y habilidades meta-cognitivas de los estudiantes (Hou, Chang, \& Sung, 2007). Para obtener resultados eficaces, es preciso que el énfasis que en la enseñanza se pone en la adquisición de logros personales se desplaze a aproximaciones más colaborativas (Liu \& Carless, 2006).

En las asignaturas de la materia de física de los grados que se imparten en l'Escola Tècnica Superior d'Enginyeria del Disseny, en la Universitat Politècnica de València (UPV), se organiza parte del Trabajo de los estudiantes en equipos. El trabajo de los equipos, formados por 6 estudiantes, consiste en la resolución de problemas, trabajo experimental en el laboratorio y redacción de informes de laboratorio. Además de elaborar documentos escritos con la resolución de problemas, éstos se presentan en clase. La evaluación de los trabajos relacionados con la resolución de problemas, tanto los documentos escritos como la presentación oral, son evaluados por otro equipo de compañeros. Los estudiantes han recibido información sobre cómo realizar la evaluación.

El presente trabajo analiza el resultado de las evaluaciones entre compañeros, llevadas a cabo durante el curso 2015-2016 en un grupo de 70 alumnos de las asignaturas Física y Electricidad, de primer curso del grado en Electrónica y Automática Industrial de la UPV, comparándolas con las hechas por dos evaluadores expertos, profesores de las asignaturas.

\section{Objetivos}

- Aprovechar que utilizamos el trabajo en equipo en la docencia de las asignaturas, y que los alumnos trabajan la comunicación efectiva con documentos escritos y presentaciones orales, para explotar las posibilidades de la evaluación entre compañeros, en un contexto de evaluación orientada al aprendizaje, como estrategia beneficiosa en la formación de los alumnos.

- Validar un sistema de evaluación entre compañeros:

- Los alumnos, en equipo, evalúan subjetivamente las presentaciones orales de los compañeros con la ayuda de una rúbrica. Lo hacen en tiempo real, al mismo tiempo que los profesores.

En trabajos anteriores hemos validado la evaluación entre compañeros de los documentos escritos (Martinez-Sala et. Al, 2015, Vidaurre et al. 2015). Se comprobó que, con la metodologia propuesta, no había diferencias significativas entre las evaluaciones hechas por profesores expertos y por equipos de alumnos. Quedaba por validar la evaluación entre compañeros de las presentaciones orales. La hipótesis de este trabajo es que, los resultados de la evaluación en equipo de las presentaciones orales de los alumnos no son significativamente diferentes a las realizadas por professores expertos.

\section{Método}

Con el fin de dar continuidad a las asignaturas de Física y de Electricidad, la composición de los equipos de trabajo de ambas asignaturas es la misma. Los equipos deben realizar seis tareas en cada una de las asignaturas, una por cada uno de los temas, que son evaluadas.

Hay dos tipos de tarea. La mitad de los equipos deben redactar un documento con la resolución de un problema, que pertenece a la colección de problemas de la asignatura, y,

(cc) EY-NC-ND 2016, Universitat Politècnica de València

Congreso In-Red (2016) 
posteriormente, deben hacer la presentación oral de este trabajo a sus compañeros en clase. La otra mitad de los equipos tiene como tarea la corrección y evaluación del documento elaborado por otro equipo (redactan un documento escrito con la evaluación siguiendo las recomendaciones de una rúbrica) y la valoración de la presentación oral correspondiente. Cada equipo hace tres tareas de resolución y tres de corrección en cada asignatura.

Las tareas se abren creando una carpeta en la plataforma de teleformación (poli[formaT] basada en Sakai). En esta carpeta se dan una serie de permisos a los alumnos para que puedan crear, leer, editar recursos propios y eliminarlos. En la carpeta se sube un documento que describe qué problema ha de hacer cada equipo y cuál ha de ser el equipo corrector. También se incluyen los plazos para subir el documento del problema, para subir las correcciones y la fecha en que han de hacer la presentación.

Los alumnos disponen de documentación sobre cómo elaborar una comunicación oral y escrita. También disponen de una rúbrica para hacer las correcciones y una guía para valorar las presentaciones,

[https://poliformat.upv.es/portal/site/GRA_12134_2015/page/95dca782-b298-4e30-91579cb233b8a356].

En las presentaciones, que realiza uno de los componentes del equipo, se valoran cinco factores: (1) lenguaje correcto y profesional, (2) orden y claridad, (3) tono de voz y énfasis, (4) utilización de medios auxiliares y (5) comunicación no verbal. Cada una de estos factores se valora con una escala de 1 a 5 , en una escala de Likert (1 Totalmente en desacuerdo, 2 En desacuerdo, $3 \mathrm{Ni}$ de acuerdo ni en desacuerdo, 4 De acuerdo, y 5 Totalmente de acuerdo).

De forma rotatoria, los componentes de los equipos deben realizar la presentación oral en clase de los problemas realizados. Una vez hecha la exposición, el equipo corrector, además de valorar la presentación con la rúbrica, es el encargado de comentarla. Los dos profesores presentes en la exposición también valoran y comentan la presentación.

Entre las dos asignaturas, Física y Electricidad, todos los alumnos tienen oportunidad de hacer una presentación oral. En el presente trabajo se analizan las valoraciones recopiladas para las 24 primeras presentaciones del curso 2015-2016.

\section{Resultados}

Se dispone de los datos de la evaluación hecha por los alumnos y por los dos profesores. La media de los dos profesores se utiliza como referencia para compararla con la evaluación de los alumnos. En una primera valoración se ha comparado el resultado global, obtenido como la media de los cinco factores analizados dándoles el valor de la escalar de Likert. En la gráfica 1 se puede observar cómo la valoración de los alumnos, en general, es superior a la asignada por los profesores, pero la mayor parte de las valoraciones tienen una diferencia inferior a un nivel en la escala de Likert. 


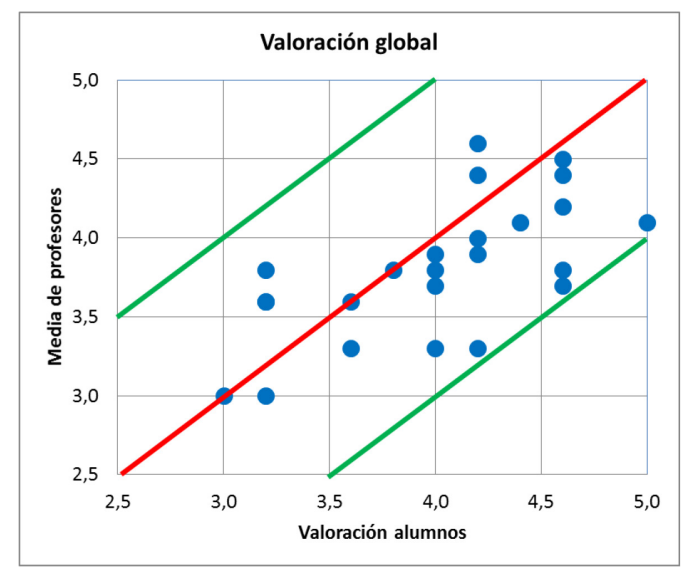

Gráfica 1: valoración global de alumnos y profesores

Para valorar los resultados, se han construido unos gráficos de Bland-Altman en donde se representa en el eje horizontal la semisuma de la valoración de profesores y alumnos en un determinado item, frente a la semidiferencia de la valoración de los alumnos y los profesores. De este modo, todo punto con un valor positivo indicará que los alumnos otorgaron una valoración superior a la media de los profesores. Adicionalmente se incluye una recta con el valor medio de los puntos que permite ver si en promedio las valoraciones han tenido una tendencia positiva o negativa. Finalmente se incluyen dos líneas de referencia en el rango de más menos dos veces la desviación típica ("límite de concordancia"), con el propósito de detectar puntos anómalos.

En la gráfica 2 se puede apreciar la comparativa de la valoración del factor "lenguaje" entre los profesores y los alumnos. En esta gráfica y en otras sucesivas, el número de puntos representados son 24 , algunos de ellos se superponen debido a que las valoraciones son discretas (de punto en punto las de los alumnos y de medio punto la media de los profesores). Cabe destacar que la línea media se encuentra muy próxima al cero, lo que indica que, aunque pudiera haber diferencias en las valoraciones, estas nunca supusieron una diferencia mayor de 1.5 puntos, y, en promedio, las valoraciones de alumnos y profesores sobre el lenguaje empleado tendían a la coincidencia.

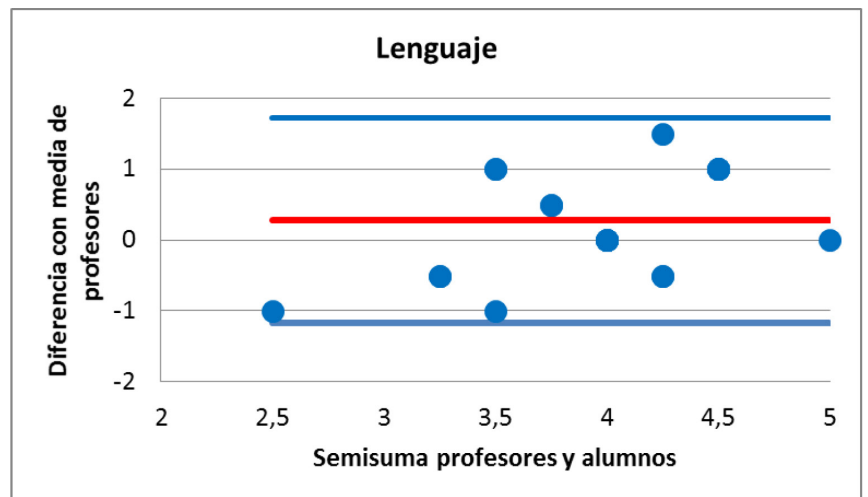

Gráfica 2: Comparativa de la valoración del lenguaje empleado por parte de los profesores y los alumnos. 
En la gráfica 3 se puede apreciar que la coincidencia era aún mayor en la valoración del factor “orden y claridad de la presentación”. Del mismo modo la dispersión era todavía menor y nunca se dieron diferencias mayores de un punto.

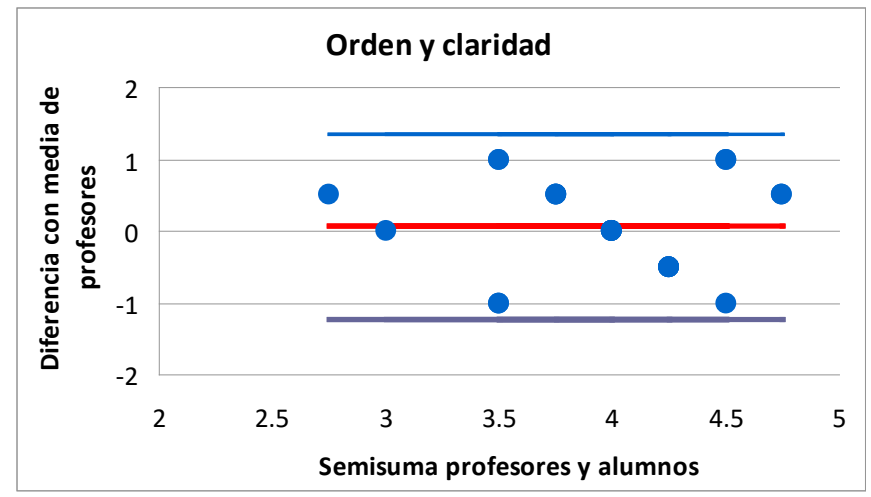

Gráfica 3: Comparativa de la valoración del orden y claridad en la exposición por parte de los profesores y los alumnos.

En la gráficas 4 se muestra la valoración del factor tono de "voz y énfasis". En este caso el valor medio de las diferencias también fue próximo a cero, lo que indica un alto grado de coincidencia. En cualquier caso, en este caso la desviación típica fue significativamente mayor, encontrándose casos con 2 puntos de diferencia. Esto pone de manifiesto que, aunque en principio la valoraciones tiendan también a ser similares, la valoración del tono de voz o el énfasis se prestan a un mayor grado de subjetividad, lo que aumenta la variabilidad de las evaluaciones.

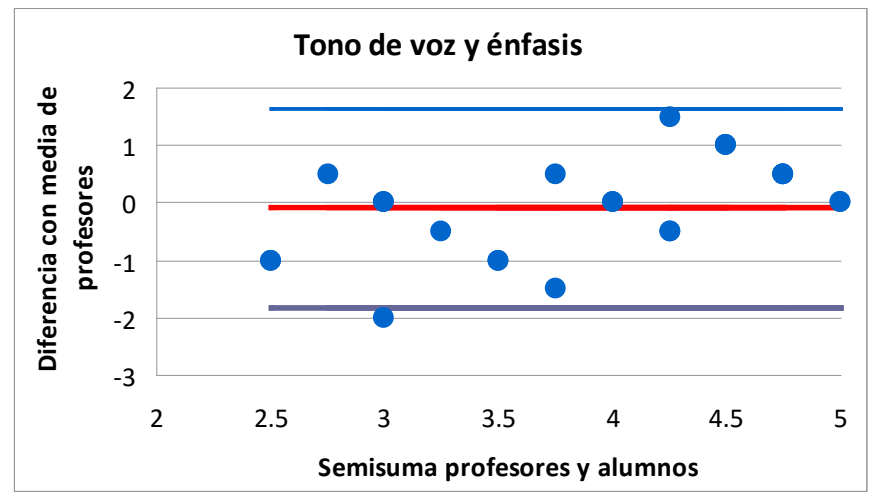

Gráfica 4: Comparativa de la valoración del tono de voz y énfasis en la exposición por parte de los profesores y los alumnos.

En la gráfica 5 se puede apreciar la comparativa de la valoración del factor "uso de medios auxiliares". En este caso, cabe resaltar que sí se aprecia cierto sesgo positivo del valor medio. Ello indica que los alumnos tendían a valorar un poco mejor el uso de medios auxiliares en comparación a cómo lo hacen los profesores. Cabe pensar, que el hecho de que los profesores estén algo más familiarizados con los medios auxiliares, les hizo tener unas expectativas más altas que los alumnos al respecto.

(cc) EY-NC-ND 2016, Universitat Politècnica de València 


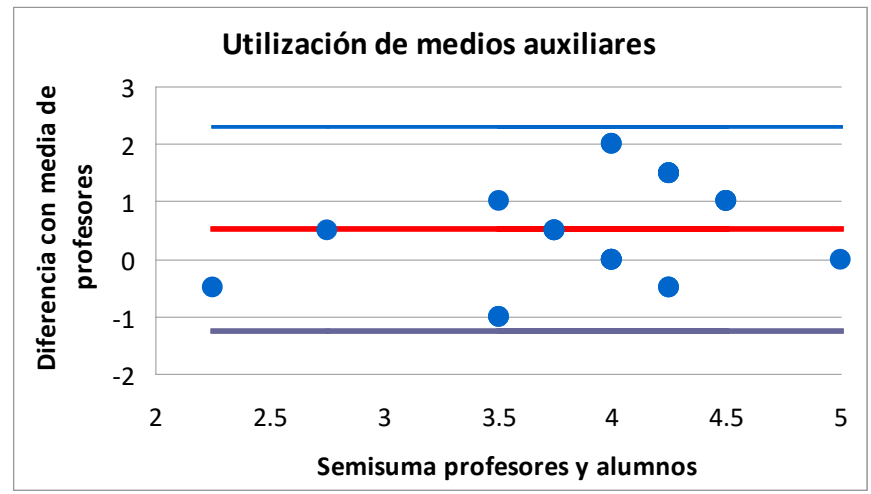

Gráfica 5: Comparativa de la valoración del uso de medios auxiliares en la exposición por parte de los profesores y los alumnos.

En la gráfica 6, se muestra la valoración del factor "comunicación no verbal". En este caso se puede apreciar que la valoración media apenas mostraba diferencias significativas y ningún valor mostró una discrepancia mayor de 1.5 puntos.

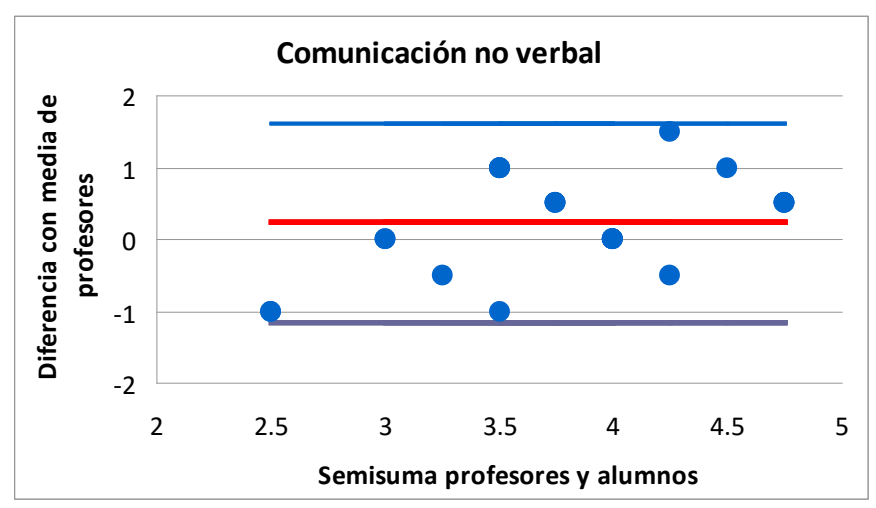

Gráfica 6: Comparativa de la valoración de la comunicación no verbal en la exposición por parte de los profesores y los alumnos.

En la gráfica 7 podemos apreciar las diferencias en valoración global ponderada de todos los items.

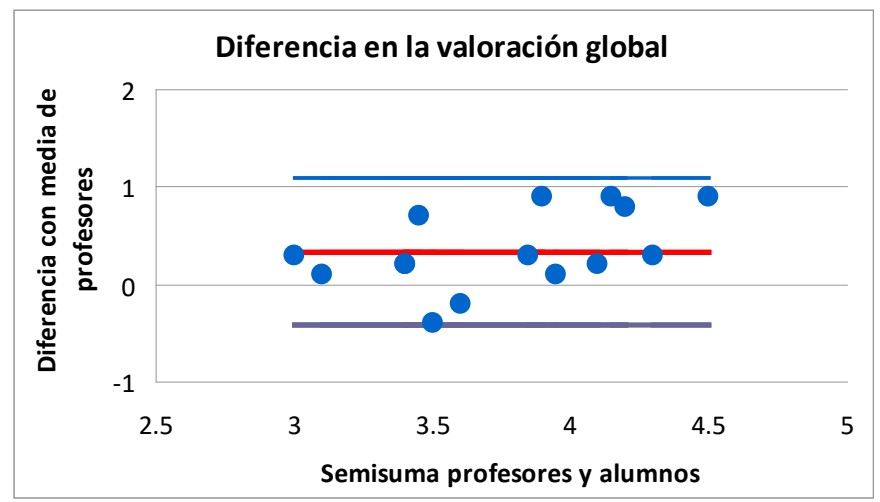

Gráfica 7: Comparativa de la valoración global en la exposición por parte de los profesores y los alumnos.

(c)) EY-NC-ND 2016, Universitat Politècnica de València

Congreso In-Red (2016) 
En la gráfica 7 se puede apreciar una gran coincidencia entre las valoraciones con una leve tendencia al alza causada, como se ha visto en la gráfica 4, por la valoración del factor referido a los medios auxiliares. También se puede apreciar claramente que, al realizar la ponderación de todos los factores, la diferencia en las valoraciones disminuye, siendo siempre menor a un punto, lo que denota una gran coincidencia en las valoraciones globales para todas las evaluaciones realizadas.

Por todo lo anterior se puede concluir que alumnos y profesores realizaban valoraciones similares, aunque en aquellos items donde la valoración puede ser más subjetiva aparecía una variabilidad más alta. Tanto los valores medios de cada categoría, como la valoración global de cada presentación, tendían a ser valoradas de manera coincidente entre profesores y alumnos más allá de un margen de incertidumbre razonable, que era siempre menor a un punto.

\subsection{Estudio de las medias de los grupos.}

Se pretende analizar si las diferencias entre las evaluaciones de los profesores y los alumnos son estadísticamente significativas

\subsubsection{Análisis de las diferencias globales.}

Se plantea si hay diferencias significativas entre la valoración global de los alumnos y la media de los profesores.

Tabla 1: análisis de valoración global

\begin{tabular}{|l|l|}
\hline Alumno/Profesor & Media global \\
\hline Alumnos: $\bar{x}(\mathrm{DE}) ; \mathrm{N}$ & $4,00(0,57) ; 24$ \\
\hline Profesor: $\bar{x}(\mathrm{DE}) ; \mathrm{N}$ & $3,72(0,54) ; 29$ \\
\hline $\mathrm{t}$ & $2,03^{\mathrm{a}}$ \\
\hline Significación estadística & $\mathrm{p}<0,05$ \\
\hline
\end{tabular}

Por lo tanto, se encuentra que hay diferencias significativas entre la valoración global de la evaluación de los alumnos y la de los profesores, con un $\alpha=0,047$. También podemos afirmar que, con el valor de $\alpha$ calculado, las diferencias no son excesivas.

\subsubsection{Análisis de las diferencias significativas factor a factor.}

Como los resultados globales no son muy contundentes se estudia si existen diferencias significativas entre profesores y alumnos factor a factor. Los resultados se muestran en la tabla.

Del análisis de los mismos podemos deducir que en cuatro de los cinco factores no se han encontrado diferencias significativas. Tan sólo en el factor "utilización de medios auxiliares" se encuentran claras diferencias significativas $(\mathrm{t}=-2,81 ; \alpha<0,01)$. 
Tabla 2: análisis factor a factor

\begin{tabular}{|l|l|l|l|l|l|}
\hline Alumno/Profesor & $\begin{array}{l}\text { 1. } \\
\text { lenguaje }\end{array}$ & $\begin{array}{l}\text { 2. orden } \mathbf{y} \\
\text { claridad }\end{array}$ & $\begin{array}{l}\text { 3. tono de } \\
\text { voz } \\
\text { énfasis }\end{array}$ & $\begin{array}{l}\text { 4. utilización } \\
\text { de medios } \\
\text { auxiliares }\end{array}$ & $\begin{array}{l}\mathbf{5 .} \\
\text { comunicación } \\
\text { no verbal }\end{array}$ \\
\hline Alumnos. $\bar{x}(\mathrm{DE})$ & $3,76(0,60)$ & $4,00(0,68)$ & $3,87(0,73)$ & $3,59(0,67)$ & $3,70(0,54)$ \\
\hline Profesor. $\bar{x}(\mathrm{DE})$ & $4,12(0,80)$ & $3,87(0,59)$ & $3,83(1,09)$ & $4,21(0,83)$ & $3,88(0,85)$ \\
\hline $\mathrm{t}$ & $-1,76^{\mathrm{a}}$ & $-0,703^{\mathrm{b}}$ & $0,135^{\mathrm{a}}$ & $-2,81^{\mathrm{a}}$ & $-0,86^{\mathrm{a}}$ \\
\hline $\begin{array}{l}\text { Significación } \\
\text { estadística }\end{array}$ & $\mathrm{p}=0,085$ & $\mathrm{p}=0,486$ & $\mathrm{p}=0,894$ & $\mathrm{p}<0,01$ & $\mathrm{p}=0,394$ \\
\hline
\end{tabular}

${ }^{a}$ Igualdad de varianzas. Se aplica el método paramétrico para aplicar t-Student

${ }^{\mathrm{b}}$ Varianzas no iguales. Se aplica el método no paramétrico para aplicar t-Student

Podemos realizar alguna hipótesis que explique la diferencia en este factor: una posibilidad es que sea el más ambiguo, que su definición no quede suficientemente aclarada para los alumnos. Otra hipótesis puede ser que los alumnos desconozcan las posibilidades de los medios auxiliares en una presentación oral. Una posible acción futura sería la revisión de la "guía del alumno", con el fin de clarificar las posibles ambigüedades, y poner en valor todos los recursos que suponen la utilización de medios auxiliares (desde la pizarra hasta los sistemas multimedia más sofisticados) en una presentación oral.

\subsubsection{Análisis de los resultados globales excluyendo el factor "Utilización de medios} auxiliares"

En la tabla 3 se muestran los resultados obtenidos prescindiendo del factor 4:

Tabla 3: análisis de valoración global excluyendo el factor "Utilización de medios auxiliares".

\begin{tabular}{|l|l|}
\hline Alumno/Profesor & Media Global sin Factor 4 \\
\hline Alumnos: $\bar{x}(\mathrm{DE}) ; \mathrm{N}$ & $3,96(0,59) ; 24$ \\
\hline Profesor: $\bar{x}(\mathrm{DE}) ;$ & $3,80(0,54) ;$ \\
\hline $\mathrm{t}$ & $-0,970^{\mathrm{a}}$ \\
\hline Significación estadística & $\mathrm{p}=0,337$ \\
\hline
\end{tabular}

En este caso no se encuentran diferencias significativas entre el valor medio de las correcciones efectuadas por los alumnos y los profesores. Podemos afirmar que, si excluimos el factor 4, no hay diferencias significativas entre los valores de las correcciones de los alumnos y los profesores.

En la representación de los diagramas de Bland-Altman de estos datos por factores, se puede ver cómo los límites de concordancia del factor 4 es el de mayor valor (gráfica 5).

Si comparamos el citado diagrama correspondiente a los valores medio con el obtenido al excluir el factor 4, se observa una reducción en el límite de concordancia del $25 \%$. Este hecho, motivado por la mayor dispersión del factor 4 , justifica que podamos prescindir del mismo al interpretar los valores medios.

(cc) EY-NC-ND 2016, Universitat Politècnica de València

Congreso In-Red (2016) 


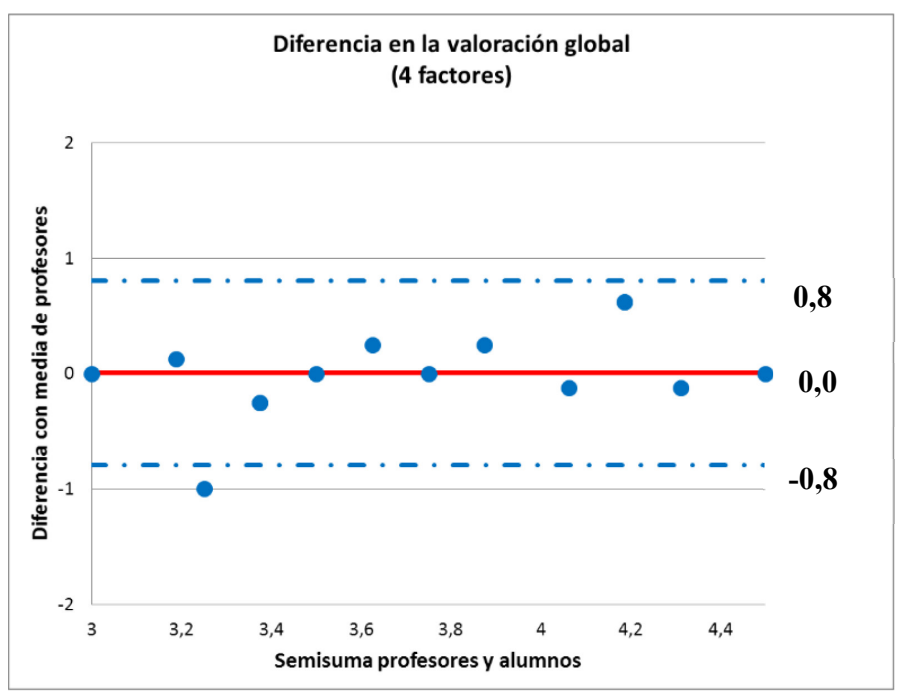

Gráfica 8: Comparativa de la valoración global en la exposición por parte de los profesores y los alumnos excluyendo el factor 4.

\section{Conclusiones}

Los alumnos pueden evaluar las presentaciones orales de sus compañeros en un contexto en el que ya evalúan documentos escritos, cuentan con guías con consejos sobre las presentaciones orales y utilizan una rúbrica sencilla.

En general, las evaluaciones hechas por los alumnos de las presentaciones orales no muestran diferencias significativas con las realizadas por los profesores.

De los cinco factores utilizados en la evaluación el factor "utilización de medios auxiliares" es el único que presenta una diferencia significativa entre la valoración de profesores y alumnos. En el resto de los factores no hay diferencia significativa entre las valoraciones.

La evaluación entre compañeros en las presentaciones orales puede considerarse como validada en el contexto definido, aunque sería conveniente mejorar la definición del factor "utilización de medios auxiliares" para que los alumnos puedan valorarlo con mayor precisión.

La metodologia propuesta de evaluación entre compañeros realizada en equipo se ha mostrado como una forma más de aprendizaje colaborativo de los estudiantes.

\section{Agradecimientos}

Los autores desean agradecer al Instituto de Ciencias de la Educación de la Universitat Politècnica de València (España) el apoyo al Grupo de Innovación Docente e-MACAFI y el apoyo financiero a través del proyecto PIME/2014/A025.

(cc) EY-NC-ND 2016, Universitat Politècnica de València 


\section{Referencias}

Black, P., \& Wiliam, D. (1998). Inside the Black Box: Raising Standards Through Classroom Assessment. Phi Delta Kappan, 80(2), 139-148. http://doi.org/10.1002/hrm

Casamayor, A., Amandi, A., \& Campo, M. (2009). Intelligent assistance for teachers in collaborative e-learning environments. Computers \& Education, 53(4), 1147-1154. http://doi.org/10.1016/j.compedu.2009.05.025

Cheng, K.-H., Hou, H.-T., \& Wu, S.-Y. (2014). Exploring students' emotional responses and participation in an online peer assessment activity: a case study. Interactive Learning Environments, 22(3), 271-287. http://doi.org/10.1080/10494820.2011.649766

Falchikov, N. (2005). Improving Assessment Through Student Involvement.

Falchikov, N., \& Goldfinch, J. (2000). Student Peer Assessment in Higher Education: A Meta-Analysis Comparing Peer and Teacher Marks. Review of Educational Research (Vol. 70). http://doi.org/10.3102/00346543070003287

Falchikov, N., \& Magin, D. (1997). Detecting Gender Bias in Peer Marking of Students' Group Process Work. Assessment \& Evaluation in Higher Education, 22(4), 385396. http://doi.org/10.1080/0260293970220403

Hou, H.-T., Chang, K.-E., \& Sung, Y.-T. (2007). An Analysis of Peer Assessment Online Discussions within a Course that uses Project-based Learning. Interactive Learning Environments, 15(3), 237-251. http://doi.org/10.1080/10494820701206974

Ibarra Sáiz, M. S., Gómez Rodríguez, G., \& Gómez Ruiz, M. Á. (2012). La evaluación entre iguales: beneficios y estrategias para su práctica en la universidad. Revista de Educación 359, (March 2016), 206-231. http://doi.org/10.4438/1988-592X-RE-2011359-092

Kaufman, J. H., \& Schunn, C. D. (2011). Students' perceptions about peer assessment for writing: Their origin and impact on revision work. Instructional Science. http://doi.org/10.1007/s11251-010-9133-6

Levine, R. E., Kelly, P. A., Karakoc, T., \& Haidet, P. (2007). Peer evaluation in a clinical clerkship: Students' attitudes, experiences, and correlations with traditional assessments. Academic Psychiatry, 31(1), 19-24. http://doi.org/10.1176/appi.ap.31.1.19

Lin, S. S. J., Liu, E. Z. F., \& Yuan, S. M. (2001). Web-based peer assessment: Feedback for students with various thinking-styles. Journal of Computer Assisted Learning, 17(4), 420-432. http://doi.org/10.1046/j.0266-4909.2001.00198.x

Liu, N.-F., \& Carless, D. (2006). Peer feedback: the learning element of peer assessment. Teaching Higher Education, 11(3), 279-290. http://doi.org/10.1080/13562510600680582

Marcoulides, G. A., \& Simkin, M. G. (1991). Evaluating student papers: The case for peer review. Journal of Education for Business, $67(2), \quad 80$. http://doi.org/10.1080/08832323.1991.10117521

Martínez-Sala R., Molina-Mateo J., Tort-Ausina I., Meseguer-Dueñas J.M.. (2015) "Peer review based evaluation in team work: students' assesment". ICERI2015. Sevilla. 980-985

Meseguer-Dueñas et al J.M.. "Guía del alumno”.

(cc) BY-NC-ND 2016, Universitat Politècnica de València

Congreso In-Red (2016) 


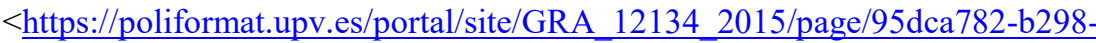
4e30-9157-9cb233b8a356>. [Consulta Marzo 2016]

Prins, F. J., Sluijsmans, D. M. a., Kirschner, P. a., \& Strijbos, J. (2005). Formative peer assessment in a CSCL environment: a case study. Assessment \& Evaluation in Higher Education, 30(4), 417-444. http://doi.org/10.1080/02602930500099219

Sargeant, J., Mann, K., Sinclair, D., Van Der Vleuten, C., \& Metsemakers, J. (2008). Understanding the influence of emotions and reflection upon multi-source feedback acceptance and use. Advances in Health Sciences Education, 13(3), 275-288. http://doi.org/10.1007/s10459-006-9039-x

Shepard, L. A. (2000). The role of assessment in a learning culture. Educational Researcher, 4-14. http://doi.org/10.3102/0013189X029007004

Tsai, C. C., \& Liang, J. C. (2009). The development of science activities via on-line peer assessment: The role of scientific epistemological views. Instructional Science, 37(3), 293-310. http://doi.org/10.1007/s11251-007-9047-0

Tsai, C.-C., Lin, S. S. ., \& Yuan, S.-M. (2002). Developing science activities through a networked peer assessment system. Computers \& Education, 38(1-3), 241-252. http://doi.org/10.1016/S0360-1315(01)00069-0

Tseng, S.-C., \& Tsai, C.-C. (2007). On-line peer assessment and the role of the peer feedback: A study of high school computer course. Computers \& Education, 49(4), 1161-1174. http://doi.org/10.1016/j.compedu.2006.01.007]

Vidaurre A., Ardid M., Cuenca V.P., Salinas I., Molina-Mateo J., Riera J., Giménez M.H., Gómez-Tejedor J.A., Martínez R., Meseguer-Dueñas J.M. (2015). "Avaluació entre companys i treball en equip" In-Red 2015. València. 1-16.

Weiss, P. R. (2000). Emotion and learning. Training \& Development, 54(11), 44-48. Retrieved from http://search.proquest.com/docview/227011307?accountid=14475

Wen, M. L., \& Tsai, C.-C. (2006). University Students' Perceptions of and Attitudes Toward (Online) Peer Assessment. Higher Education, 51(1), 27-44. http://doi.org/10.1007/s10734-004-6375-8 